\title{
Poster: Teaching Letter Writing using a Programmable Haptic Device Interface for Children with Handwriting Difficulties
}

\author{
Younhee Kim; Zoran Duric’’ Naomi Lynn Gerber* \\ George Mason University
}

\author{
Arthur R. Palsbo , Susan E. Palsbođl \\ OBSLAP RESEARCH, LLC
}

\begin{abstract}
We designed a virtual hand-writing teaching system for children with handwriting difficulties due to attention or motor deficits, using a haptic interface that could provide a neutral, repetitive engaging approach to letter writing. The approach we took to accomplish this included: (a) Using letter primitives, (b) User friendly interface for teachers, therapists, subjects and parents, (c) Adjustable force and assessment mode, and (d) Quantitative reports. We evaluated 4 subjects. We obtained pre-training letter formation, then followed with a prescribed training session in which a fixed number of haptic driven repetitions was performed. Subjects were post-tested with free-form letter writing. Anecdotally, 2 children had obvious improvement in accuracy of letter formation, one slowed down speed with which he formed letters, which resulted in more legible handwriting. Children were engaged in the process and reported they had fun and would do it again.
\end{abstract}

\section{INTRODUCTION}

Learning to write is considered a fundamental requirement for literacy and for integration into society. Many children have difficulty learning to write and/or write legibly. These children are usually taught using one-to-one training, using a labor intensive approach. Teachers and parents have expressed interest in a device driven, game-like, neutral method for teaching letters and handwriting. Haptic technology has features that are likely to provide these elements. Several research groups [3, 9, 8, 10, 4] have developed hand-writing systems using a haptic interface, and recently the positive effect of haptic training sessions have been reported [2, 6].

We designed a virtual letter-writing using primitives and focused on teaching system for children with motor and attention deficits (ADD) using a haptic interface (applied for U.S. Provisional Patent) [7]. Since the user domain is different from previous letterwriting teaching systems, we focused on increasing the usability of the program, and providing an evaluation the student's competence. The approach we took to accomplish this included: (a) Using letters primitives, (b) User friendly interface for therapists, subjects and parents, (c) Adjustable force and assessment mode (d) Quantitative report of outcome. We conducted experiments with four children with motor deficit or ADD and the results will be presented in the Results section.

\section{Primitives for a letter library}

While other letter-writing approaches record a teacher's movement and use it as a template for a student to follow, we used letter primitives and created letters using them. The advantages of the primitives are that (a) we can create any letter from the primitives and that

\footnotetext{
*e-mail: ykim9@gmu.edu

†e-mail: zduric@cs.gmu.edu

† -mail: ngerber1@gmu.edu

$\S$ e-mail: obslap.research@ comcast.net

Ile-mail: spalsbo@gmu.edu
}

(b) the shape of letters can be easily modified by changing scaling and rotation parameters.

The basic primitives are a straight line, circle, and ellipse. We can generate the primitives using the mathematical equations and the trajectories of writing all letters consists of straight lines, pen lifts (ellipse), and arcs of a circle. Due to limited space, we skip the description of the equations, but we show the example of the letter using the primitives in Figure 1 instead.

We used an existing therapeutic intervention, Handwriting Without Tears [5], as a template for letter formation. We implemented the trajectories of letters following the textbook instructions including the order of strokes and shape of the letters based on stereotypical movements of line (up-stroke, down-stroke, cross) lift-off, ellipse, partial or full circles. For example, a letter "A", is a straight line stroke followed by pen-lift-ellipse, second straight line, penlift-ellipse, and the third straight line.

We customized the pen-lift height to permit children with motor deficits to perform the task. The pen-lifts are marked in red and the letters are marked in blue.
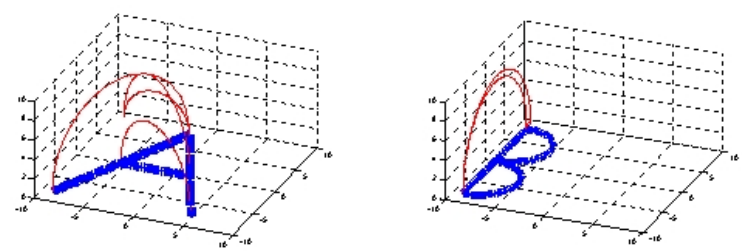

Figure 1: Example of letter trajectories using primitives.

\section{SYSTEM DESCRIPTION}

We developed a letter-writing system using a haptic device, the Phantom Omni [1] model. The Omni model has $160 \times 120 \times 70 \mathrm{~mm}$ workspace and the maximum force is $3.3 \mathrm{~N}$. We implemented our program on the MathWorks MATLAB, Simulink and Handshake proSENSE Virtual Touch Toolbox development platform.

The haptic can provide both visual and auditory cues for the task, providing immediate feedback. This is helpful for those who learn well with auditory stimulation. We programmed an auditory prompt, for example, to help start the process, "Get Ready" and "Go" (Figure 2). The trainer can adjust parameters such as the haptic speed and the number of repetitions for training. And also more importantly, these parameters can be changed easily. Our system incorporated variable force capability. Force could be adjusted up or down as the child demonstrates more competence. The treatment approach was to repeat the task until it was quickly, accurately and efficiently performed. One could then test the activity with no force to determine how well the task had been learned and performed. This approach tests bottom up learning; with motor, sensory and repetitive force. The learning can thus be customized to the student's condition and optimized for better learning. The report captures the time, error rate in percentage and the strategy used to perform the task. For example, whether it follows the prescribed path, or stops/starts frequently or overdraws the line. 


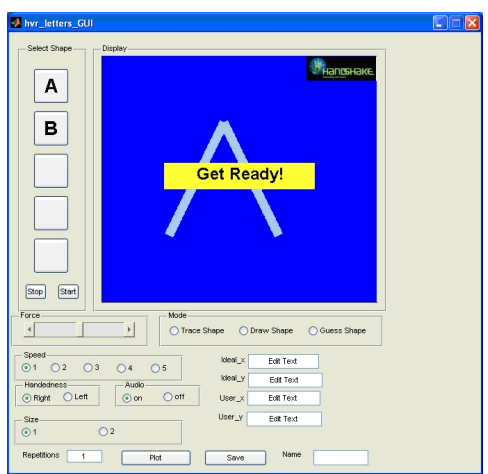

Figure 2: Our program user interface.

Table 1: Demographics

\begin{tabular}{ccccc} 
Subject \# & Sex & Age & Motor Deficit & Attention Deficit \\
\hline 1 & M & 10 & N & Y \\
2 & M & 9 & N & Y \\
3 & M & 12 & Y & N \\
4 & M & 12 & N & N
\end{tabular}

\section{EXPERIMENTAL RESULTS}

We obtained George Mason IRB approval to test the haptic in children who has attended a summer camp which was convened to address handwriting difficulties. After obtaining informed consent from the parent and the assent from the child, the subjects were placed in front of the haptic, given instructions about what they were to do to permit the haptic to guide their making of the letters, and observed. To evaluate the system, we asked the subject to write the letter before training and after training in free hand using a usual pen and we observed the change of the letter. We conducted trials with four subjects. The subject's information is presented in Table 1.

\subsection{Procedure}

1. The subject is asked to write the letter "A" and "B" in the usual fashion, using a standard pencil.

2. The subject is introduced to the haptic and the test procedure is described.

3 . The subjects grasps the haptic stylus and positions the hand comfortably, permitting the haptic to guide the formation of the letter. This is repeated 5 times, with each event receiving a verbal cue "get ready" from the program.

4. After completing the training the subjects were asked to redraw the letters, which were visually compared to the earlier attempt.

\subsection{Results}

The letters written before training and after training were recorded and staff was similarly asked whether there were objective changes in letter writing pre/post training session. The results are shown in Table 2. Anecdotally, 2 children had obvious improvement in accuracy of letter formation and one slowed down speed with which he formed letters helping his legibility. Figure 3 presents letter writing made by subject 2 pre-training and post-training. It is of some interest that all subjects enjoyed the session. Three of the participants indicated that the construction of the haptic pen was confining and limited the freedom of movement of the arm, making the letter writing different from what they would do if they were writing.
Table 2: Investigator Assessment

\begin{tabular}{cl} 
Subject \# & Change from Pre-Post session \\
\hline 1 & Improved letters (more careful, slower) \\
2 & Significant Improvement in Letter B \\
3 & Improved \\
4 & No Change
\end{tabular}

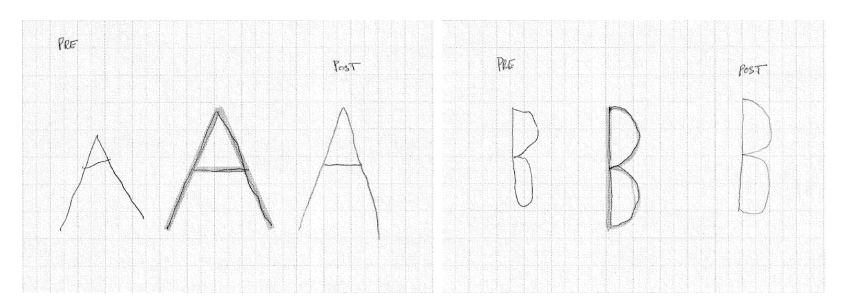

Figure 3: Letter writing comparison between pre training and post training using standard pencil and paper.

\section{CONCLUSION}

In this paper, a hand-writing training system using a haptic device is presented for children with handwriting difficulties. In our system, letters are generated using primitives and the performance is reported in a quantitative manner. Visual and audio cues are used to increase the user feedback. We evaluated four children with motor deficit or ADD. We compared pre-training with post-training free form letter writing. Their performance was improved in writing letters after a prescribed training session in which a fixed number of repetitions were performed. The results would show that the potential advantage of 3D force feedback over the 2D force feedback that can be attained in classrooms. However, further work including more quantitative and statistical evaluation will be needed to show being superior of the 3D force feedback.

\section{REFERENCES}

[1] Refer to the Sensable products at www.sensable.com.

[2] J. Bluteau, S. Coquillart, Y. Payan, and E. Gentaz. Haptic guidance improves the visuo-manual tracking of trajectories. PLOS ONE, 3(3):e1775, Mar 2008.

[3] K. Henmi and T. Yoshikawa. Virtual lesson and its application to virtual calligraphy system. In Proceedings of the IEEE International Conference on Robotics and Automation, 1998.

[4] Y. K. Kim and X. Yang. Hand-writing rehabilitation in the haptic virtual environment. In Proceedings of the IEEE International Workshop on Haptic Audio Visual Environments and their Applications, 2006.

[5] J. Z. Olsen. Handwriting without tears.

[6] R. Palluel-Germain, F. Bara, A. H. de Boisferon, B. Hennion, P. Gouagout, and E. Gentaz. A visuo-haptic device - telemaque increases kindergarten children's handwriting acquisition. In $W H C$, pages 72-77, 2007.

[7] S. E. Palsbo, A. R. Palsbo, S. Johnson, N. L. Gerber, Y. Kim, Z. Duric, W. Norblad, and M. Hopkins. Fine-motor execution using repetitive force-feedback. U.S. Provisional Patent, Application No. 12/153903, 2008.

[8] S. Saga, N. Kawakami, and S. Tachi. Haptic teaching using opposite force presentation. In Proceedings of the world haptics conference, 2005.

[9] J. Solis, C. A. Avizzano, and M. Bergamasco. Teaching to write japanese characters using a haptic interface. In Proceedings of the 10th Symposium on Haptic Interfaces for Virtual Environment and Teleoperator Systems, 2002.

[10] C. Teo, E. Burdet, and H. Lim. A robotic teacher of chinese handwriting. In Proceedings of the 10th Symposium on Haptic Interfaces for Virtual Environment and Teleoperator Systems, 2002. 